\title{
Les gènes programmeurs du développement
}

L'identification et la caractérisation de gènes contrôlant les étapes très précoces du développement embryonnaire constituent une des découvertes majeures des dernières années.

\section{Bruno Jarry \\ Professeur à l'Université Louis- Pasteur de Strasbourg}

Yves Grau

Chercheur au Cnrs

\section{ADRESSE}

B. Jarry et Yves Grau : laboratoire de génétique de l'Inserm, faculté de médecine, 11, rue dernier. Cette approche, fructueuse

de l'Inserm, faculté de médecine, 11, rue dernier. Cette approche, fructueuse chez les microorganismes, a été appliquée aux processus du développement de la drosophile et a conduit à l'identification d'un grand nombre de gènes.

La drosophile possède dans certains tissus des chromosomes géants. Ces chromosomes ont une morphologie particulière, constituée de bandes sombres et de bandes claires alternées dans un arrangement caractéristique et constant d'un animal à l'autre. Les généticiens ont depuis longtemps utilisé ces bandes comme marqueurs cytologiques pour dresser la carte des gènes. Ces cartes ont été correllées avec les cartes génétiques obtenues par l'analyse des fréquences de recombinaison entre mutations et il est aujourd'hui courant d'identifier la position d'un gène donné sur un chromosome par le numéro de la bande correspondante Cette particularité s'est révélée extrêmement utile pour la génétique moléculaire. Elle permet d'apprécier directement de quelle région du chromosome provient un segment d'ADN cloné et par conséquent de se passer complètement de toute information biochimique préalable sur les produits du gène. Supposons que l'on ait cloné un segment d'ADN. Ce segment contient, par définition, sur ses deux chaînes, la même séquence de bases, la même information génétique que celle qui est présente dans la région particulière du génome dont il est issu. Il est techniquement facile de traiter des cellules contenant des chromosomes géants, de telle sorte que les molécules d'ADN se dénaturent. Si l'on dépose sur cette préparation une goutte de solution contenant le segment d'ADN cloné préalablement dénaturé, ses deux 


\section{ERRATUM}

\section{La légende de la figure 1 de l'article de Bruno JARRY et Yves GRAU, page 249, est erronnée.}

\section{Vous auriez dû lire :}

Figure I. L'hybridation in situ.

La glande salivaire (I), prélevée chez la larve, contient de très grandes cellules. Ces cellules (2) possèdent des chromosomes géants qui sont composés de plusieurs centaines de molécules d'ADN juxtaposées (3). Ces molécules (4) sont dénaturées par un traitement chimique. La sonde, dont quelques atomes sont radioactifs est mise au contact des molécules dénaturées (5). Les brins de la sonde vont s'hybrider aux molécules d'ADN du chromosome par appariement moléculaire (6). Après un lavage pour éliminer la sonde qui n'a pas réagi, seules les molécules de sondes qui ont retrouvé leur partenaire exact restent accrochées sur les chromosomes (7). 


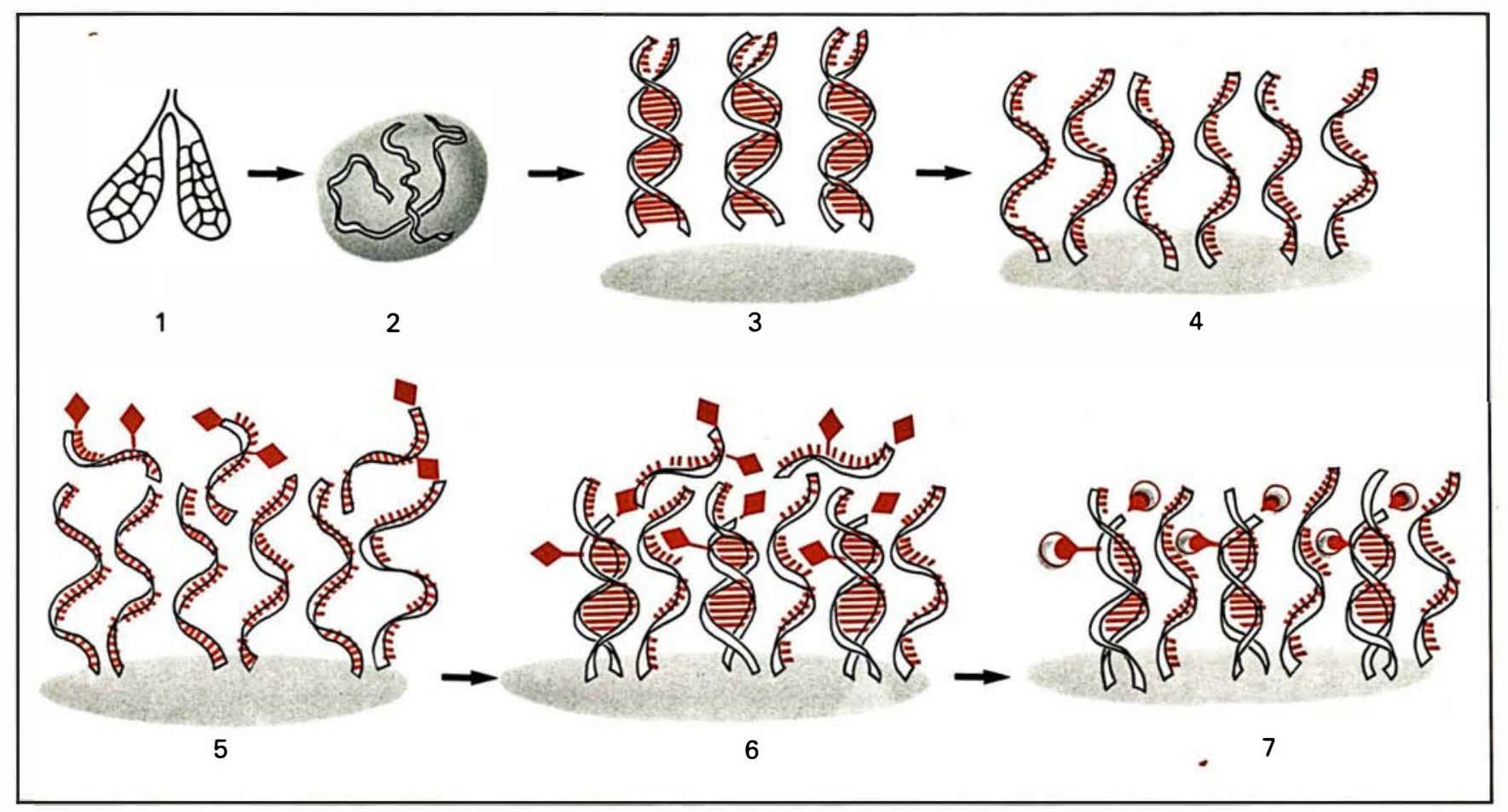

Figure 1. Structure chimique des principaux antisécrétoires gastriques commercialisés ou en cours d'études sur l'homme.

Cimétidine, ranitidine et famotidine sont des anti-H2; seul le premier peut être considéré comme un inhibiteur de structure de l'histamine car possédant le noyau imidazole. La pirenzépine est un dérivé des tricycliques antidépresseurs. L'oméprazole fait partie de la série des benzimidazoles, mais n'est probablement actif que par les métabolites auxquels il donne naissance à l'intérieur de la cellule pariétale acido-sécrétrice.

brins reconnaîtront la région précise du chromosome dont ce segment est la copie et s'y fixeront par appariement moléculaire (figure 1). Le segment cloné peut lui-même être repéré parce qu'il a été marqué par un isotope radioactif. Il est alors aisé d'observer directement sous le microscope, le site d'appariement du segment d'ADN cloné sur le chromosome (figure 2, voir p. suivante). Cette technique a reçu le nom d'hybridation in situ. De par son principe même, elle permet d'établir une relation directe entre le phénotype et le génotype. La génétique classique, couplée à la cytogénétique, apporte la double information de l'identité du gène - révélée par l'expression de son phénotype - et de sa localisation sur la carte au niveau d'une bande particulière du chromosome. La génétique moléculaire et l'hybridation in situ vont permettre d'établir la relation $m / s n^{\circ} 5$ septembre 85 directe entre les segments d'ADN clonés et la structure du chromosome. C'est la première étape dans le clonage et la définition moléculaire d'un gène.

L'analyse systématique de nombreuses mutations de l'ADN a montré qu'une fraction importante des mutations spontanées chez la drosophile provient de l'insertion dans le gène, d'éléments génétiques particuliers appelés éléments transposables. Ces éléments ont été clonés et bien caractérisés au niveau moléculaire; quelques uns ressemblent de fait beaucoup aux rétrovirus que l'on trouve intégrés dans le génome des mammifêres. Le repérage de leur site d'insertion sur la carte de restriction de la région chromosomique permet de définir le site de la mutation. Une information du même ordre est obtenue en analysant l'ADN d'une souche mutante où la mutation est due à la cassure d'un chromosome. Dans ce cas aussi, la carte physique de l'ADN est fortement modifiée par la mutation et l'identification du point de cassure permet de définir la séquence qui contient le gène dont la fonction a été altérée par la mutation. C'est l'application de ces techniques aux gènes programmeurs du développement qui a permis l'explosion de résultats à laquelle nous assistons aujourd'hui.

\section{Le développement de la drosophile}

Après la fécondation, l'œuf se développe comme un syncitium de noyaux totipotents. La plupart des noyaux migrent ultérieurement à la périphérie de l'œuf et vont former après la cellularisation une couche monocellulaire, le blastoderme, qui entoure les réserves nutritives de l'embryon. Ce stade précoce corres- 


\section{REFERENCES}

1. Anderson $\mathrm{K} V$, Nüsslein-Volhard C. Information for the dorsal-ventral pattern of Drosophila embryo is stored as maternal mRNA. Nature $1984 ; 311: 223-7$.

2. Steward R, McNally F J, Schedl P. Isolation of the dorsal locus of Drosophila. Nature 1984; 311: 262.

3. Sanchez-Herrero E, Vernos I, Marco R, Morata G. Genetic organization of Drosophila bithorax complex. Nature 1985; 313: 108-13.

4. Lawrence P A, Morata G. The elements of the bithorax complex. Cell 1983; 35 : 595-601.

5. Lewis E B. A gene complex controlling segmentation in Drosophila. Nature 1978; $276: 565-70$.

6. Bender W, Akam M, Karch F, et al. Molecular genetics of the bithorax complex in Drosophila melanogaster. Science 1983; 221 : 23-9.

7. North G. Cloning the genes that specify fruit flies. Nature 1983; 303: 134-6.

8. Beachy P A, Helfand S L, Hogness D S. Segmental distribution of bithorax complex proteins during Drosophila development. Nature $1985 ; 313: 545-51$

9. Brahic M, Haase A. Detection of viral sequences of low reiteration frequency by in situ hybridization. Proc Natl Acad Sci USA 1978; $75: 6125-30$.

10. Akam M. The location of ultrabithorax transcripts in Drosophila tissue section. Embo 7 $1983 ; 2: 2075-84$.

11. White R A H, Wilcox M. Protein products of the bithorax complex in Drosophila. Cell 1984; $39: 163-71$.

12. Gehring W. The homeo box: a key to the understanding of development? Cell 1985; $40: 3-5$.

13. Stent G. Thinking in one dimension. The impact of molecular biology on development. Cell $1985 ; 40: 1-2$.

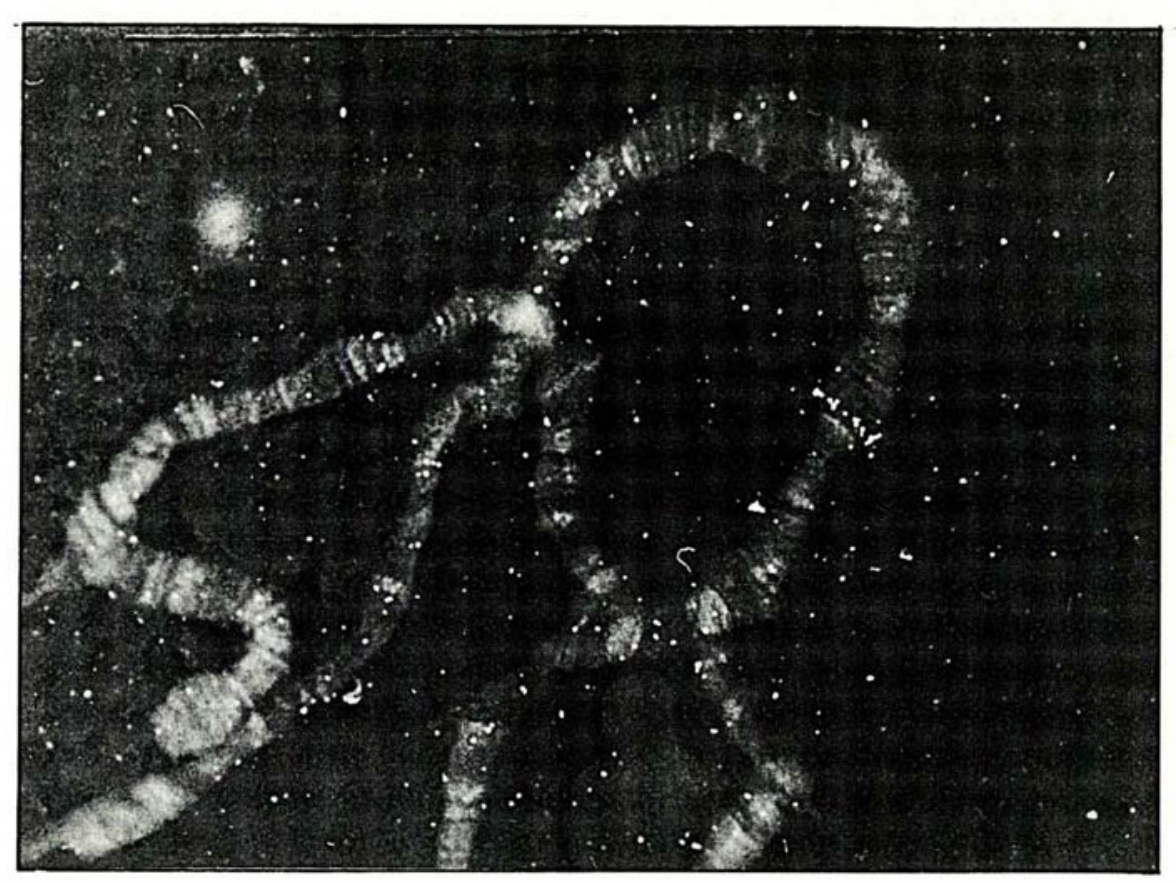

Figure 2. L'identifïcation d'un segment d'ADN cloné par hybridation in situ. Le segment est fixé sur une bande particulière du chromosome. La radioactivité qui lui est associée réduit les grains d'argent d'une émulsion photographique appliquée sur la préparation. Les grains indiquent très précisément le site correspondant à l'hybridation de la sonde.

pond à une étape cruciale dans le développement de la drosophile. La détermination, c'est-à-dire la programmation irréversible du plan général du corps, est mise en place. Elle se manifeste en fin d'embryogenèse par la différenciation des segments. Cette organisation en métamères, unités de base répétées, est un processus fondamental du développement de la plupart des organismes supérieurs. A ce stade, chaque segment est composé de deux classes de cellules aux avenirs très différents. Une classe de cellules forme les tissus de la larve; la deuxième classe de cellules forme le second organisme qui émergera de la carcasse de la larve à la métamorphose, la mouche adulte. Ces cellules sont groupées en paquets appelés les disques imaginaux. Elles se'multiplient pendant toute la vie larvaire mais ne se différencient que lors de la métamorphose. Le développement à partir du blastoderme aboutit à la formation de 14 segments qui se retrouveront jusque chez l'adulte : au moins trois seg- ments céphaliques, trois segments thoraciques $\left(T_{1}, T_{2}, T_{3}\right)$ et huit segments abdominaux ( $A_{1}$ à $\left.A 8\right)$. Ce même stade du développement est marqué également par le début de la synthèse de l'ARN. Quelle est l'origine de l'information nécessaire à cette détermination?

\section{Polarité de l'embryon}

Les premiers gènes qui interviennent pour réguler le développement embryonnaire sont ceux qui déterminent la polarité des axes de l'œuf : antéro-postérieur et dorsoventral. Ces gènes ont été définis par des mutations qui ne modifient l'organisation de l'embryon que sur un seul axe, sans changer l'assemblage des structures le long de l'autre. Ils sont actifs bien avant la fertilisation, au cours de l'ovogenèse. Pour cette raison, on les appelle des gènes à " action maternelle ".

C'est la mise en place de la polarité dorso-ventrale qui a été étudiée jusqu'à présent avec le plus de détails. Onze gènes différents ont été locali- 
sés sur les chromosomes. Les mutations correspondantes conduisent à des embryons chez lesquels manquent les structures ventrales et latérales. L'ensemble de l'embryon n'est plus composé que de structures dorsales. Toutes ces mutations exprimant le même phénotype, il est vraisemblable que les produits de ces gènes font tous partie du même mécanisme de création de la polarité, chaque gène spécifiant un des éléments particuliers du processus. Certains de ces mutants "dorsalisés " peuvent être corrigés en injectant dans l'embryon anormal de l'ARN préparé à partir d'embryons normaux [1]. On est donc raisonnablement sûr qu'un signal chimique spécifique, ARN ou protéine, joue le rôle de substance morphogénétique à l'intérieur de l'œuf. Tout se passe comme si les différents composants de l'œuf s'organisaient en fonction de la concentration locale de ce signal. Un de ces gènes, le gène dorsal, a été cloné l'année dernière [2]. L'ARN correspondant est présent dans les ovaires maternels et dans le très jeune embryon mais disparait dans les stades ultérieurs. Le temps est maintenant proche où le décryptage biochimique de cette information de position, et des processus de reconnaissance qu'elle implique, pourra être entrepris. Ces gènes maternels définissent le plan général du corps. Le choix des programmes de développement des cellules est le fait d'autres fonctions génétiques : les gènes homéotiques.

\section{De la répétition à la diversité}

Il existe des drosophiles mutantes avec des phénotypes extravagants : des ailes, par exemple, sortent de la tête à la place des yeux (la mutation ophtalmoptera), des pattes à la place des antennes (la mutation Antennapedia) ou des ailes à la place des balanciers (la mutation bithorax). Ces mutations, qui transforment une partie du corps en structures appartenant à une autre partie du corps sont appelées homéotiques. A ce jour, plus d'une quinzaine de gènes homéotiques ont été identifiés dans le génome. Souvent ces gènes sont localisés l'un à côté de l'autre sur les chromosomes définissant un complexe, c'est-à-dire un groupe de gènes adjacents présentant des relations fonctionnelles, mais séparables par recombinaison méiotique. L'un d'entre eux, le complexe bithorax est localisé sur le bras droit du troisième chromosome et contient au moins neuf gènes homéotiques. L'analyse génétique [3] a permis de le diviser en trois domaines : Ultrabithorax ( $U b x)$, abdominal-A (abd$A)$ et Abdominal-B $(A b d-B)$. Le domaine $U b x$ contient au moins trois types de mutations récessives viables, bithorax $(b x)$, bithoraxoid $(b x d)$ et postbithorax $(p b x)$ qui représentent des fonctions largement indépendantes.

A quoi servent ces fonctions? Suivons l'analyse génétique : les mouches normales ont une paire d'ailes qui appartiennent au segment thoracique 2 (T2), et une paire de balanciers appartenant au segment throracique $3\left(\mathrm{~T}_{3}\right)$. Chaque segment est formé de deux compartiments, l'un antérieur l'autre postérieur. Construisons un individu homozygote pour un allèle mutant de $b x$. Cette mouche présente un phénotype caractéristique où le compartiment antérieur des balanciers est transformé en compartiment antérieur de l'aile; les compartiments postérieurs eux n'ont pas changé. Cette mouche possède donc une paire et demie d'ailes, mais seulement une demie paire de balanciers. Le tissu alaire formé dans les balanciers de la mouche mutante est en tout point semblable à celui des ailes. La mutation $b x$ provoque donc la transformation du compartiment antérieur $\mathrm{T}_{3}$ en structure caractéristique du compartiment antérieur T2. L'interprétation la plus simple de cette observation est que la fonction spécifiée par le gène $b x^{+}$est requise pendant la croissance des cellules du compartiment antérieur du balancier : la fonction venant à manquer chez le mutant, ces cellules sont aiguillées vers un autre devenir et se développent comme si elles se trouvaient dans le compartiment antérieur $\mathrm{T}_{2}$ en donnant du tissu alaire. Le corollaire de ce raisonnement est que la fonction $b x^{+}$n'est pas active dans le compartiment antérieur T2. Dans cet exemple, la fonction $b x^{+}$agit comme un processus d'aiguillage binaire entre deux voies du développement. Tous les éléments du complexe bithorax fonctionnent de la même manière en déterminant le devenir des compartiments ou des segments de la mouche adulte d'une façon précise et logique : une mouche homozygote mutante $p b x / p b x$ aura le compartiment postérieur $\mathrm{T}_{3}$ transformé en compartiment postérieur T2, une mouche homozygote mutante $b x d / b x d$ le compartiment antérieur du segment abdominal 1 transformé en compartiment antérieur $\mathrm{T}_{3}$ et ainsi de suite. La présence du produit de ces gènes homéotiques est nécessaire "dès le stade du blastoderme et tout au long de l'ontogenèse des disques imaginaux [4].

On sait maintenant que les gènes homéotiques sont responsables aussi de la diversité des segments embryonnaires. Il existe une autre classe de mutations récessives dans le complexe bithorax : leurs effets ne sont jamais observés sur la mouche adulte parce que les mutants homozygotes meurent à la fin de l'embryogenèse. Les différents segments se sont différenciés et présentent des structures morphologiques caractéristiques. Si le complexe bithorax est entièrement délété, les larves meurent en différenciant la tête, le segment thoracique $T_{1}$, le segment thoracique $T_{2}$, puis un deuxième segment thoracique $T_{2}$, un troisième $T_{2}$ et ainsi de suite. Nous voilà revenus à l'arthropode ancestral composé d'une succession de segments tous identiques! En d'autres termes, il n'existe plus chez cet individu de distinction entre segments thoraciques et segments abdominaux, le second segment thoracique apparaissant comme la structure morphologique de base.

En étudiant ainsi l'effet d'autres délétions [3], cette fois-ci partielles du complexe, on arrive à une décomposition en trois domaines : le domaine $U b x$ qui contrôle la diversification thoracique, le domaine $a b d-A$ qui contrôle le développement des segments abdominaux $\mathrm{A}_{1}$ à $\mathrm{A}_{4}$ et enfin le domaine $A b d-B$ celui des segments abdominaux $\mathrm{A}_{5}$ à A8. Chaque domaine contient un certain nombre de fonc- 


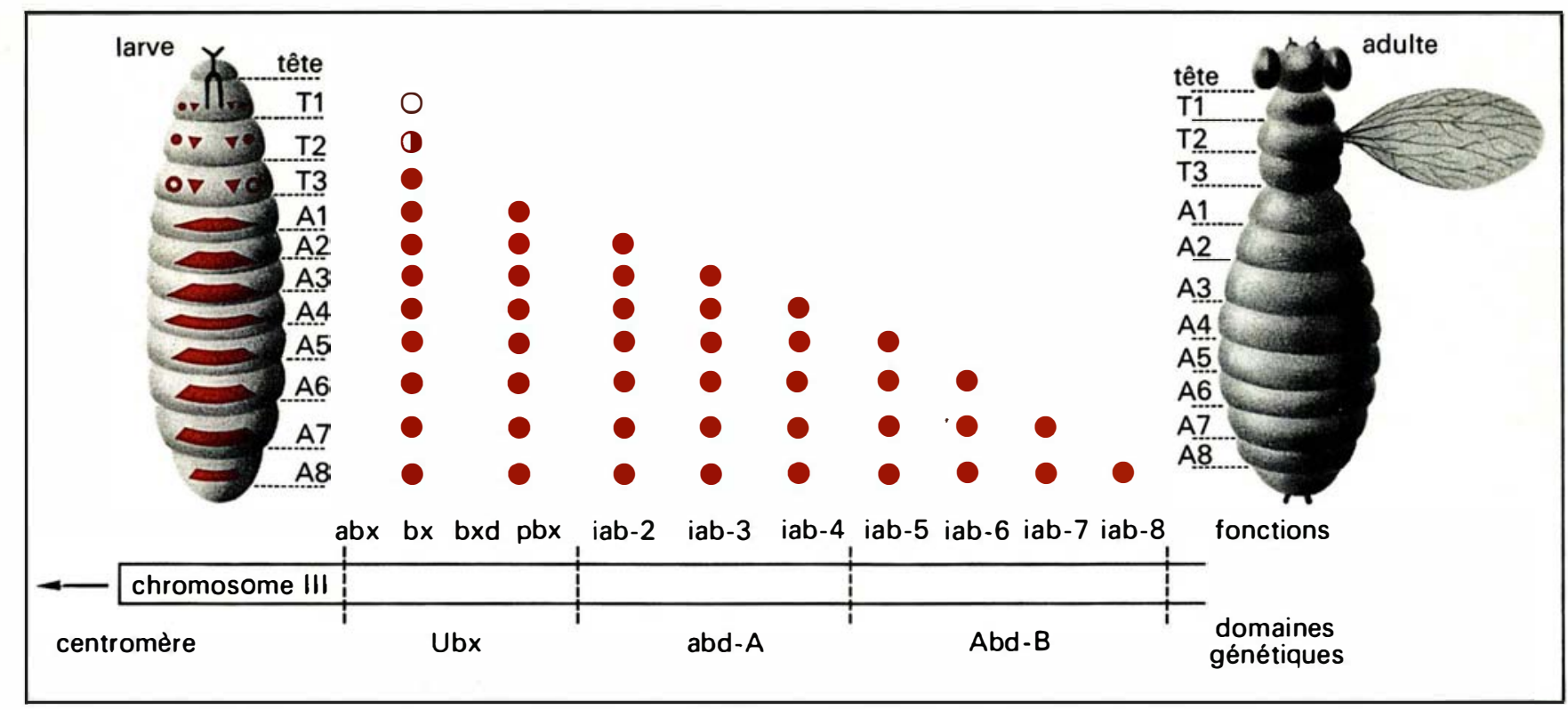

Figure 3. Domaines génétiques et fonctions du complexe bithorax. Le complexe bithorax est subdivisé en trois domaines génétiques (Ubx, abd-A, Abd-B) qui correspondent aux unités de complémentation. Par exemple, la perte du domaine Ubx conduit à l'absence des fonctions $\mathrm{abx}, \mathrm{bx}, \mathrm{bxd}, \mathrm{pbx}$. Le domaine fonctionnel $d u$ complexe s'étend du compartiment postérieur du segment thoracique $T_{2}$ au segment abdominal A8. La première fonction du complexe (abx) s'exprime dans le compartiment postérieur du segment $T_{2}$ (cette fonction est limitée seulement à l'aire ventrale de ce compartiment). Puis, de compartiment en compartiment, une nouvelle fonction est pas encore si les fonctions abdominales (iab2 à iab8) s'expriment à l'intérieur d'un compartiment ou d'un segment. On aboutit ainsi au segment abdominal A8 dans lequel tout le complexe est activé. La transcription pourrait se faire de façon hiérarchique : pour obtenir la morphologie caractéristique d'un segment abdominal donné, il faut que les fonctions homéotiques spécifiant le développement des segments plus antérieurs soient activées en plus de l'activation de l'élément clé, la fonction spécifique de ce segment. Il n'y a pas d'élément actif dans les segments antérieurs. Ce sont d'autres gènes homéotiques (notamment ceux du complexe Antennapedia) qui spécifient les voies de développement des segments antérieurs.

tions qui sélectionnent le développement des compartiments en leur donnant une étiquette génétique comme nous l'avons vu plus haut pour le domaine $U b x$. Ces observations ont conduit E. Lewis [5] à proposer que plus un segment est postérieur dans l'animal, plus nombreuses seront les fonctions génétiques du complexe qui seront exprimées (figure 3). Ce modèle, probablement trop simpliste [3], suggère qu'il existe une correspondance topologique entre l'ordre génétique des fonctions homéotiques et la séquence des compartiments contrôlés.

En conclusion, les gènes homéotiques sont les meilleurs candidats comme éléments génétiques gouvernant la réalisation des voies de développement. Ils révèlent ainsi l'existence d'un véritable programme inscrit dans les chromosomes. Les produits de ces gènes interagissent de façon concertée pour sélectionner le destin des cellules en contrôlant l'activité transcriptionnelle des gènes de structures responsables de la morphologie finale.

\section{Biologie moléculaire du complexe bithorax}

L'ADN compris entre les bandes $89 \mathrm{E}_{1}-\mathrm{E}_{4}$ des chromosomes géants a été cloné sous forme de segments partiellement chevauchants [6].
L'ensemble couvre d'un seul tenant 100000 paires de bases. La quasitotalité des mutations conduisant aux phénotypes associés au domaine Ubx (figure 4) y ont été localisées. Chacune de ces mutations est causée soit par l'insertion d'un élément transposable, soit par une cassure du chromosome. Deux unités génétiques y ont été repérées [7]. La première mesure 75000 bases de long et constitue l'unité $U b x^{+}$; la seconde, l'unité $b x d^{+}, 20000$ bases. La taille de cette première unité est beaucoup plus importante que celle d'un gène classique. Curieusement, les autres fonctions du domaine, $b x^{+}$et $a b x^{+}$sont localisées à l'intérieur de l'unité $U b x^{+}$et la fonction $p b x^{+}$à l'intérieur de l'unité $b x d^{+}$. Ces unités génétiques importantes ne codent finalement que pour des ARN de petite taille $(4700,4300$ et 3200 bases). Les ARN de l'unité Ubx sont composés pour la plus grande part de séquences localisées sur l'ADN aux deux extrémités de l'unité. Ils apparaissent d'une façon légèrement décalée au début de l'embryogenèse, et sont formés par l'épissage différentiel entre les séquences des extrémités et quelques micro-exons, encore mal définis pour certains, localisés à l'intérieur de l'unité.

Pour expliquer l'interpénétration d'éléments génétiques qui donnent naissance à des produits de gène de 
fonctions similaires mais différentes, D. Hogness et al. postulent l'existence de plusieurs schémas d'épissage qui permettraient, à partir du grand prémessager de 75000 bases, de produire différents ARN contenant une partie constante et une partie variable. Ces ARN seraient transcrits ou épissés de façon régulée au cours du développement de telle manière qu'un type moléculaire particulier s'accumule dans les cellules des différents compartiments. Un de ces ARN au moins contient l'information codante pour une protéine [8].

L'unité bithoraxoid $\left(b x d^{+}\right)$code également pour plusieurs types de molécules d'ARN. D'une façon de nouveau inattendue, leur séquence contient de très nombreux triplets non-sens dans les trois phases de lecture, et ne code probablement pas pour une protéine.

\section{Vers une embryologie moléculaire}

Une nouvelle technique mise au point sur des tissus infectés par des virus [9] permet de franchir un pas supplémentaire. Il s'agit d'une application de l'hybridation moléculaire, que nous avons évoquée plus haut, mais où cette fois-ci la sonde radioactive est appariée non pas à l'ADN des chromosomes, mais aux molécules d'ARN qui sont dans les cellules. On apprécie ainsi simultanément sous le microscope la morphologie des cellules sur la coupe et la concentration en ARN spécifique transcrit d'un gène donné à l'intérieur de ces mêmes cellules. On crée donc un pont entre l'embryologie classique qui étudie les interactions entre les cellules dans l'ensemble de l'organisme et la génétique moléculaire qui définit les produits de gènes présidant à ces interactions. Appliquée aux messagers de l'unité $U b x^{+}[10]$, cette technique révèle qu'au cours des premiers stades de développement de l'embryon, ces ARN sont très abondants dans les cellules des segments postérieurs, principalement dans les ganglions nerveux, mais absents de celles des segments les plus antérieurs. Dans les disques imaginaux, les résultats sont plus clairs encore : l'ARN $U b x$ n'a pas pu être détecté dans les cellules des disques du segment thoracique $\mathrm{T}_{2}$, mais il est par contre abondant dans les disques du segment $\mathrm{T}_{3}$.

Ces résultats ont été confirmés et étendus à l'étude de la localisation dans les cellules, des protéines codées par ces ARN messagers $[8,11]$. Une de ces protéines, purifiée à partir de bactéries programmées avec un fragment d'ADN de l'unité $U b x^{+}$, a servi à préparer un anticorps qui reconnaît la famille des protéines $U b x^{+}$. Cet anticorps, appliqué sur des sections d'embryon, détecte faiblement la présence de son antigène dans la région postérieure $T_{2}$, fortement dans la région postérieure $\mathrm{T}_{3}$ et antérieure $A_{1}$, la concentration de l'antigène allant en décroissant dans les portions antérieures $\mathrm{A}_{2}$ à $\mathrm{A}_{7}$. De même, les cellules des disques imaginaux des ailes et de la deuxième paire de pattes ne contiennent pas d'antigène alors qu'il est très abondant dans les disques imaginaux de la troisième patte et du balancier. Les molécules d'antigène sont localisées pour la plus grande partie dans le noyau des cellules et par conséquent au contact direct du matériel génétique.

Les prédictions du modèle de Lewis sont en partie vérifiées au niveau cellulaire et moléculaire. Il n'en reste pas moins que les mécanismes qui doivent fonctionner pour effec-

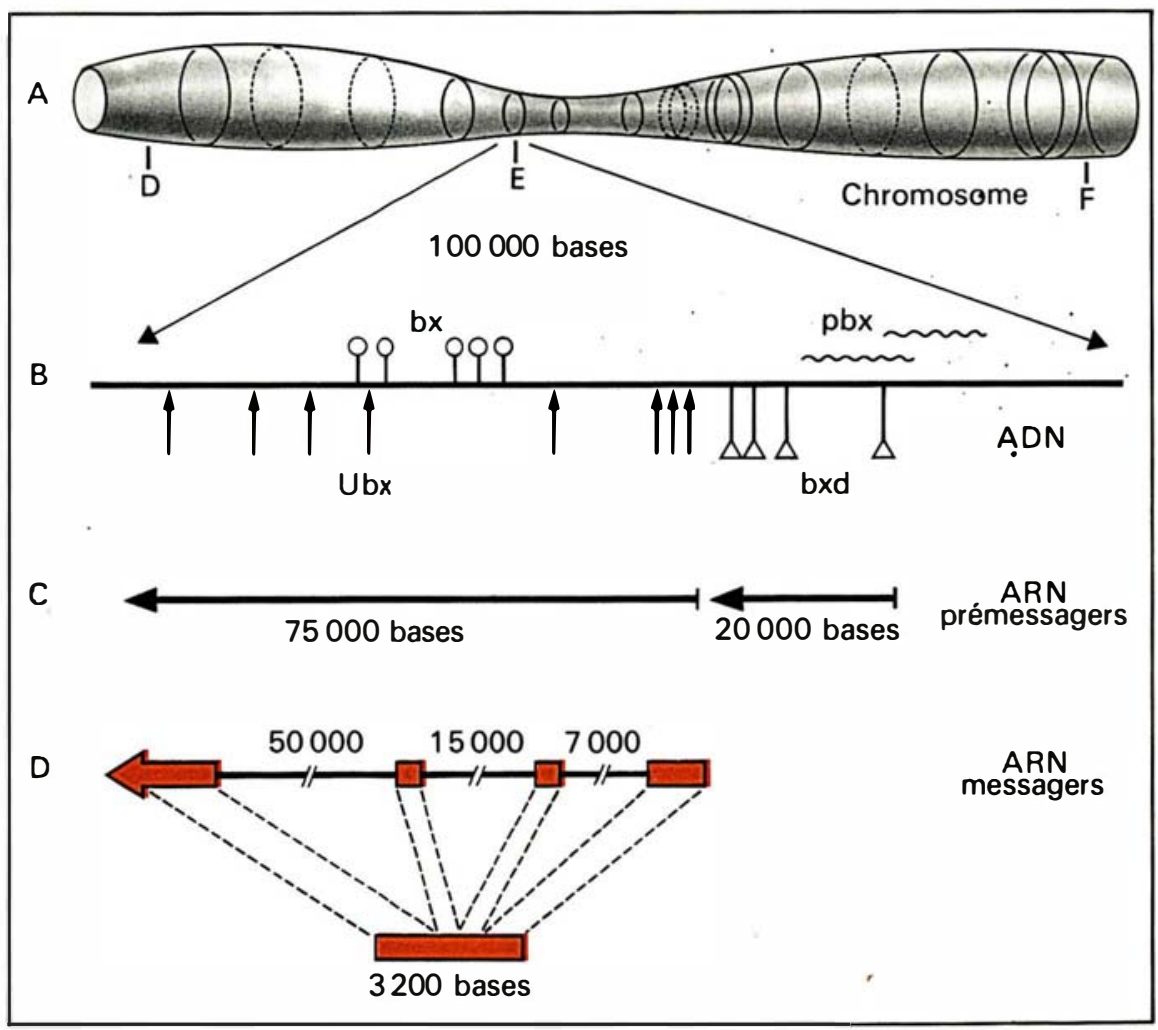

Figure 4. Carte physique de l'ADN dans la région $89 \mathrm{D}-\mathrm{F}$ du troisième chromosome.

A Schéma du chromosome géant. B Sites d'intégration des éléments transposables ou de cassure $d u$ chromosome dans la région du complexe bithorax. Les mutations marquiées $\uparrow$ donnent le phénotype ultrabithorax, les mutations 9 le phénotype bithorax, les mutations $\Delta$ le phénotype bithoraxoid; enfin les mutations marquées sont de courtes délétions et donnent le phénotype parabithorax. C Les unités de transcription de l'ARN dans la région du domaine ultrabithorax. D L'épissage de l'ARN prémessager de l'unité ultrabithorax. Trois types d'ARN messagers différents ont été caractérisés. Les réactions différentielles d'épissage sont probablement régulées en fonction du temps et dans des cellules différentes. 
tuer une répartition si fine de l'expression génétique n'en sont pas pour autant clarifiés. Cependant, nous disposons de quelques indices glanés au cours de l'étude de la structure des gènes homéotiques.

Les trois domaines du complexe possèdent en commun une région répétée codant pour soixante acides aminés [12]. Cet enchaînement polypeptidique, baptisé "homéo box ", se retrouve conservé presque intact dans d'autres gènes homéotiques, mais aussi incorporé dans des protéines particulières d'un tout autre organisme, la levure. Ces protéines sont localisées elles aussi dans le noyau et contrôlent, d'une façon encore incomplètement élucidée au niveau moléculaire, les processus de différenciation dans cette espèce. Gehring a suggéré que le domaine protéique codé par l'" homéo box" puisse entrer en contact direct avec l'ADN du chromosome et permettre ainsi aux protéines homéotiques de contrôler directement l'activité des gènes qui doivent réaliser la différenciation en jouant le rôle de répresseur ou d'activateur de l'expression génétique dans son ensemble. Cette hypothèse est bien sûr fortement inspirée des modèles de contrôle de l'expression des gènes chez les bactéries.

\section{Conc/usion}

I'analyse des mécanismes héréditaires qui permettent l'élaboration ordonnee de tout organisme multicellulaire ne fait encore que commencer. En soixante ans, l'embryologie classique nous a donné le détail de la construction des différentes ébauches tissulaires et de l'agencement de ces tissus à l'intérieur du corps de nombreux organismes. Ce travail, essentiellement descriptif, a conduit à la classification du règne animal sur des critères embryologiques précis qui mettent en évidence la grande diversité des situations et des moyens utilisés pour organiser le développement dans les différentes espèces. Il n'en reste pas moins vrai que la théorie de l'évolution et l'universalité du mécanisme de stockage de l'information au niveau de l'ADN, qui préside fondamentalement au développement, nous autorisent à rechercher un modèle général.

$\mathrm{Au}$ cours des dernières années, l'approche génétique a permis de découvrir quelques éléments qui, ayant été retrouvés dans des organismes aussi éloignés dans la classification que la mouche drosophile et le ver nématode (qui n'a pas été décrit ici), sont probablement parmi les plus fondamentaux. On sait aujourd'hui comment des restrictions précises se manifestent au niveau cellulaire bien avant que des différences claires ne deviennent morphologiquement apparentes. La détermination des cellules n'est pas liée à la structure histologique à laquelle elles appartiennent, mais seulement à la place qu'occupait dans l'embryon la ou les cellules fondatrices de la lignée.

On peut toutefois s'interroger sur la généralité de ce modèle [12]. Il est bien établi, par exemple, que chez les mammifêres le continuum embryologique est différent de celui trouvé chez les insectes. Jusqu'à récemment, aucun mutant homéotique n'y avait été décrit. Il semble toutef ois que cette lacune vienne d'être comblée. Le mutant rachiterata de la souris, chez lequel la septième vertèbre cervicale ne portant pas de paire de côtes est remplacée par la première vertèbre thoracique qui en porte une paire, pourrait être le premier exemple d'un tel phénotype. Reste bien sûr à le démontrer formellement au niveau moléculaire. Ceci pourrait être facilité par une autre découverte du groupe de Gehring. Ces chercheurs ont en effet identifié des séquences "homéo box "dans le génome d'organismes aussi différents de la drosophile que la grenouille, la souris ou l'homme [12]. Vestiges de l'évolution ou mécanismes fondamentaux conservés au-delà de la barrière des espèces? Il ne faudra plus très longtemps pour savoir si les gènes de souris clonés en utilisant les séquences " homéo box" de drosophile comme sonde possèdent les mêmes propriétés fonctionnelles. Quoiqu'il en soit, même si plusieurs schémas de programmes de développement ont pu être mis en place de façon concurrente dans divers organismes [13], la connaissance intime de l'un d'eux sera fondamentale pour notre compréhension de la vie.

\section{Summary}

The genetic analysis of the development of drosophila has led to the concept of a developmental program written in the DNA. This analysis has allowed to define the key steps in the activation of this set of instructions. Such studies shed light on the old problem of embryology: how is made the choice of the development pathways. There are processes that allow a cell to recognize its position within a morphogenetic field. This results in the choice of specific modules of the developmental program for each cell, although this cell possesses the whole program. The modules have been identified and their function is to control the positioning of the animal's different structures.

\section{TIRES A PART}

B. Jarry : laboratoire de génétique moléculaire des eucaryotes du Cnrs, unité U. 184 de l'Inserm, faculté de médecine, 11 , rue Humann, 67085 Strasbourg Cedex. 